\title{
SECOND MAIN THEOREMS WITH WEIGHTED COUNTING FUNCTIONS AND ALGEBRAIC DEPENDENCE OF MEROMORPHIC MAPPINGS
}

\author{
SI DUC QUANG
}

(Communicated by Franc Forstneric)

\begin{abstract}
The purpose of this article is twofold. The first is to prove a new second main theorem for meromorphic mappings and moving hyperplanes of $\mathbb{P}^{n}(\mathbb{C})$, where the counting functions are truncated multiplicities and have different weights. Our result is an extension of previous second main theorems for moving hyperplanes with the truncated (to level $n$ ) counting functions. As its application, the second purpose of this article is to prove a new algebraic dependence theorem for meromorphic mappings having the same inverse images of some moving hyperplanes, where the moving hyperplanes involve the assumption with different roles.
\end{abstract}

\section{INTRODUCTION}

Nevanlinna theory for meromorphic mappings of $\mathbb{C}^{m}$ into the complex projective space $\mathbb{P}^{n}(\mathbb{C})$ intersecting a finite set of fixed hyperplanes or moving hyperplanes in $\mathbb{P}^{n}(\mathbb{C})$ was started about 70 years ago and has grown immensely. The main goal in this theory is establishing the second main theorem, which is an estimate of the characteristic function of the mappings by the sum of some counting functions. For the case of the mappings intersecting fixed hyperplanes, the second main theorem given by Cartan and Nochka [1,3] is sharp. For the case of meromorphic functions and small functions, i.e., $n=1$, the sharp result is given by Yamanoi [16]. So far, there is no sharp second main theorem for meromorphic mappings into general projective spaces intersecting moving hyperplanes with truncated counting functions. We state here some recent available results. Firstly, we recall the following.

Let $a_{1}, \ldots, a_{q}(q \geq n+1)$ be $q$ meromorphic mappings of $\mathbb{C}^{m}$ into the dual space $\mathbb{P}^{n}(\mathbb{C})^{*}$ with reduced representations $a_{i}=\left(a_{i 0}: \cdots: a_{i n}\right)(1 \leq i \leq q)$. We say that $a_{1}, \ldots, a_{q}$ are located in general position if $\operatorname{det}\left(a_{i_{k} l}\right) \not \equiv 0$ for any $1 \leq i_{0}<i_{1}<\cdots<i_{n} \leq q$. Let $\mathcal{M}_{m}$ be the field of all meromorphic functions on $\mathbb{C}^{m}$. Denote by $\mathcal{R}\left(\left\{a_{i}\right\}_{i=1}^{q}\right) \subset \mathcal{M}_{m}$ the smallest subfield which contains $\mathbb{C}$ and all $\frac{a_{i k}}{a_{i l}}$ with $a_{i l} \not \equiv 0$.

For the case of nondegenerate meromorphic mappings of $\mathbb{C}^{m}$ into $\mathbb{P}^{n}(\mathbb{C})$ intersecting moving hyperplanes, the first second main theorem with truncated (to level $n$ ) counting functions was given by $\mathrm{Ru}[8$ for the case $m=1$ and reproved for the

Received by the editors December 5, 2015.

2010 Mathematics Subject Classification. Primary 32H30, 32A22; Secondary 30D35.

Key words and phrases. Nevanlinna, second main theorem, meromorphic mapping, moving hyperplane. 
general case by Thai-Quang [10]. For the case of degenerate meromorphic mappings, in 9] $\mathrm{Ru}$ and Wang gave a second main theorem for moving hyperplanes with counting function truncated to level $n$. Following that, the result of $\mathrm{Ru}$-Wang was improved by Thai-Quang [11] and Quang-An [7]. Recently, in [5] the author improved and extended all those results to the following.

Theorem A (see [5, Theorem 1.1a]). Let $f: \mathbb{C}^{m} \rightarrow \mathbb{P}^{n}(\mathbb{C})$ be a meromorphic mapping. Let $\left\{a_{i}\right\}_{i=1}^{q}(q \geq 2 n-k+2)$ be meromorphic mappings of $\mathbb{C}^{m}$ into $\mathbb{P}^{n}(\mathbb{C})^{*}$ in general position such that $\left(f, a_{i}\right) \not \equiv 0(1 \leq i \leq q)$, where $k+1=\operatorname{rank}_{\mathcal{R}\left\{a_{i}\right\}}(f)$. Then the following assertion holds:

$$
\| \frac{q}{2 n-k+2} T_{f}(r) \leq \sum_{i=1}^{q} N_{\left(f, a_{i}\right)}^{[k]}(r)+o\left(T_{f}(r)\right)+O\left(\max _{1 \leq i \leq q} T_{a_{i}}(r)\right) .
$$

Here, by the notation " $\mid P$ " we mean that assertion $P$ holds for all $r \in[0, \infty)$ outside a Borel subset $E$ of the interval $[0, \infty)$ with $\int_{E} d r<\infty$.

We see that in the above result, the counting functions $N_{\left(f, a_{i}\right)}^{[k]}(r)$ involve the inequality with the same roles. My aim in this paper is to consider the case where the truncated counting functions involve the second main theorem with different weights. Namely, we will extend the above result to the following.

Theorem 1.1. Let $f: \mathbb{C}^{m} \rightarrow \mathbb{P}^{n}(\mathbb{C})$ be a meromorphic mapping. Let $\left\{a_{i}\right\}_{i=1}^{q}(q \geq$ $2 n-k+2)$ be meromorphic mappings of $\mathbb{C}^{m}$ into $\mathbb{P}^{n}(\mathbb{C})^{*}$ in general position such that $\left(f, a_{i}\right) \not \equiv 0(1 \leq i \leq q)$. Assume that $k+1=\operatorname{rank}_{\mathcal{R}\left\{a_{i}\right\}}(f)$. Let $\lambda_{1}, \ldots, \lambda_{q}$ be $q$ positive numbers with $(2 n-k+2) \max _{1 \leq i \leq q} \lambda_{i} \leq \sum_{i=1}^{q} \lambda_{i}$. Then the following assertions hold:

$$
\| \frac{\sum_{i=1}^{q} \lambda_{i}}{2 n-k+2} T_{f}(r) \leq \sum_{i=1}^{q} \lambda_{i} N_{\left(f, a_{i}\right)}^{[k]}(r)+o\left(T_{f}(r)\right)+O\left(\max _{1 \leq i \leq q} T_{a_{i}}(r)\right) .
$$

We see that Theorem A is a corollary of Theorem 1.1 when $\lambda_{1}=\cdots=\lambda_{q}$.

As an application of this second main theorem, in the last section we will prove an algebraic dependence theorem for meromorphic mappings sharing moving hyperplanes regardless of multiplicities. To state our result, we need the following.

Let $f_{i}: \mathbb{C}^{m} \rightarrow \mathbb{P}^{n}(\mathbb{C})(1 \leqslant i \leqslant \lambda)$ be meromorphic mappings with reduced representations $f_{i}:=\left(f_{i 0}: \cdots: f_{\text {in }}\right)$. Let $a_{i}: \mathbb{C}^{m} \rightarrow \mathbb{P}^{n}(\mathbb{C})^{*}(1 \leqslant i \leqslant q)$ be slowly moving hyperplanes in general position with reduced representations $a_{i}:=\left(a_{i 0}\right.$ : $\left.\cdots: a_{i n}\right)$. Assume that $\left(f_{t}, a_{i}\right):=\sum_{j=0}^{n} f_{t j} a_{i j} \neq 0$ for each $1 \leq t \leq \lambda, 1 \leq i \leq q$ and $\left(f_{1}, a_{i}\right)^{-1}\{0\}=\cdots=\left(f_{\lambda}, a_{i}\right)^{-1}\{0\}$. Put $A_{i}=\left(f_{1}, a_{i}\right)^{-1}\{0\}$. Assume that every analytic set $A_{i}$ has the irreducible decomposition as follows: $A_{i}=\bigcup_{j=1}^{t_{i}} A_{i j}$. Set $A=\bigcup_{A_{i j} \not A_{k l}}\left\{A_{i j} \cap A_{k l}\right\}$ with $1 \leq j \leq t_{i}, 1 \leq l \leq t_{k}, 0 \leq i, k \leq q-1$.

Denote by $T[n+1, q]$ the set of all injective maps from $\{1, \cdots, n+1\}$ to $\{1, \cdots, q\}$. For each $z \in \mathbb{C}^{m} \backslash\left\{\bigcup_{\beta \in T[n+1, q]}\left\{z \mid a_{\beta(1)}(z) \wedge \cdots \wedge a_{\beta(n+1)}(z)=0\right\} \cup A \cup \bigcup_{i=1}^{\lambda} I\left(f_{i}\right)\right\}$, we define $\rho(z)=\sharp\left\{j \mid z \in A_{j}\right\}$. Then $\rho(z) \leq n$. Indeed, suppose that $z \in A_{i}$ for each $0 \leq i \leq n$. Then $\sum_{j=0}^{n} f_{1 j}(z) \cdot a_{i j}(z)=0$ for each $0 \leq i \leq n$. Since $a_{\beta(1)}(z) \wedge \cdots \wedge a_{\beta(n+1)}(z) \neq 0$, it implies that $f_{1 i}(z)=0$ for each $0 \leq i \leq n$. This means that $z \in I\left(f_{1}\right)$. This is impossible. 
For any positive number $r>0$, define $\rho(r)=\sup \{\rho(z)|| z \mid \leq r\}$, where the supremum is taken over all $z \in \mathbb{C}^{m} \backslash\left\{\bigcup_{\beta \in T[n+1, q]}\left\{z \mid g_{\beta(1)}(z) \wedge \cdots \wedge g_{\beta(n+1)}(z)=\right.\right.$ $\left.0\} \cup A \cup \bigcup_{i=1}^{\lambda} I\left(f_{i}\right)\right\}$. Then $\rho(r)$ is a decreasing function. Let

$$
d:=\lim _{r \rightarrow+\infty} \rho(r)
$$

Then $d \leq n$. If for each $i \neq j, \operatorname{dim}\left\{A_{i} \cap A_{j}\right\} \leq m-2$, then $d=1$.

In 2001, Ru [8] gave an algebraic dependence theorem for meromorphic mappings sharing several moving hyperplanes as follows.

Theorem B (see [8, Theorem 1]). Let $f_{1}, \cdots, f_{\lambda}: \mathbb{C}^{m} \rightarrow \mathbb{P}^{n}(\mathbb{C})(\lambda \geq 2)$ be nonconstant meromorphic mappings. Let $a_{i}: \mathbb{C}^{m} \rightarrow \mathbb{P}^{n}(\mathbb{C})^{*}(1 \leq i \leq q)$ be slowly moving hyperplanes in general position. Assume that $\left(f_{i}, a_{j}\right) \not \equiv 0$ and $\left(f_{1}, a_{j}\right)^{-1}\{0\}=\cdots=\left(f_{\lambda}, a_{j}\right)^{-1}\{0\}$ for each $1 \leq i \leq \lambda, 1 \leq j \leq q$. Denote $A_{j}=\left(f_{1}, a_{j}\right)^{-1}(\{0\})$. Let $l$ be a positive integer with $2 \leq l \leq \lambda$. Assume that for each $z \in A_{j}(1 \leq j \leq q)$ and for any $1 \leq i_{1}<\cdots<i_{l_{j}}<\bar{q}, f_{i_{1}}(z) \wedge \cdots \wedge f_{i_{l}}(z)=0$. If $q>\frac{d \lambda n^{2}(2 n+1)}{\lambda-l+1}$, then $f_{1}, \cdots, f_{\lambda}$ are algebraically dependent over $\mathbb{C}$, i.e., $f_{1} \wedge \cdots \wedge f_{\lambda} \equiv 0$ on $\mathbb{C}^{m}$.

After that, the result of Ru has been improved and extended by Thoan-Duc and Quang in [12, 13] and [6] when the number of moving hyperplanes is reduced. We see that in the above result, the number $l$ is fixed for all moving hyperplanes. Now we will consider the case where the number $l$ depends on the moving hyperplanes. It means that for each $j$, we suppose that there exists a positive number $l_{j}\left(l_{j}\right.$ may be $+\infty)$ such that $f_{i_{1}} \wedge \cdots \wedge f_{i_{l_{j}}}$ on $A_{j}$ for any $l_{j}$ mappings. Also, we will try to reduce the number $q$ of moving hyperplanes. Namely, we will prove the following.

Theorem 1.2. Let $f_{1}, \cdots, f_{\lambda}: \mathbb{C}^{m} \rightarrow \mathbb{P}^{n}(\mathbb{C})(\lambda \geq 2)$ be nonconstant meromorphic mappings. Let $a_{i}: \mathbb{C}^{m} \rightarrow \mathbb{P}^{n}(\mathbb{C})^{*}(1 \leq i \leq q)$ be slowly moving hyperplanes in general position. Assume that $\left(f_{i}, a_{j}\right) \not \equiv 0$ and $\left(f_{1}, a_{j}\right)^{-1}\{0\}=\cdots=\left(f_{\lambda}, a_{j}\right)^{-1}\{0\}$ for each $1 \leq i \leq \lambda, 1 \leq j \leq q$. Denote $A_{j}=\left(f_{1}, a_{j}\right)^{-1}(\{0\})$. Let $l_{1}, \ldots, l_{q}$ be $q$ positive integers with $2 \leq l_{i} \leq \lambda$. Assume that for each $z \in A_{j}(1 \leq j \leq q)$ and for any $1 \leq i_{1}<\cdots<i_{l_{j}}<q, f_{i_{1}}(z) \wedge \cdots \wedge f_{i_{l_{j}}}(z)=0$. If $q>\frac{d \lambda\left(n^{2}+2 n\right)+\sum_{j=1}^{q} l_{j}}{\lambda+1}$, then $f_{1}, \cdots, f_{\lambda}$ are algebraically dependent over $\mathbb{C}$, i.e., $f_{1} \wedge \cdots \wedge f_{\lambda} \equiv 0$ on $\mathbb{C}^{m}$.

Letting $l_{1}=\cdots=l_{q}=l$, we get the following corollary.

Corollary 1.3. Let $f_{1}, \cdots, f_{\lambda}: \mathbb{C}^{m} \rightarrow \mathbb{P}^{n}(\mathbb{C})(\lambda \geq 2)$ be nonconstant meromorphic mappings. Let $a_{i}: \mathbb{C}^{m} \rightarrow \mathbb{P}^{n}(\mathbb{C})^{*}(1 \leq i \leq q)$ be slowly moving hyperplanes in general position. Assume that $\left(f_{i}, a_{j}\right) \not \equiv 0$ and $\left(f_{1}, a_{j}\right)^{-1}\{0\}=\cdots=\left(f_{\lambda}, a_{j}\right)^{-1}\{0\}$ for each $1 \leq i \leq \lambda, 1 \leq j \leq q$. Denote $A_{j}=\left(f_{1}, a_{j}\right)^{-1}(\{0\})$. Let $l$ be a positive integer with $2 \leq l \leq \lambda$. Assume that for each $z \in A_{j}(1 \leq j \leq q)$ and for any $1 \leq i_{1}<\cdots<i_{l}<q, f_{i_{1}}(z) \wedge \cdots \wedge f_{i_{l}}(z)=0$. If $q>\frac{d \lambda\left(n^{2}+2 n\right)}{\lambda-l+1}$, then $f_{1}, \cdots, f_{\lambda}$ are algebraically dependent over $\mathbb{C}$, i.e., $f_{1} \wedge \cdots \wedge f_{\lambda} \equiv 0$ on $\mathbb{C}^{m}$.

Letting $d=1, \lambda=l=2$ in Corollary 1.3 we get the following uniqueness theorem for meromorphic mappings sharing moving hyperplanes. 
Corollary 1.4. Let $f_{1}, f_{2}: \mathbb{C}^{m} \rightarrow \mathbb{P}^{n}(\mathbb{C})$ be nonconstant meromorphic mappings. Let $a_{i}: \mathbb{C}^{m} \rightarrow \mathbb{P}^{n}(\mathbb{C})^{*}(1 \leq i \leq q)$ be slowly moving hyperplanes in general position. Assume that $\left(f_{i}, a_{j}\right) \not \equiv 0$ and

(i) $\min \left\{1, \nu_{\left(f_{1}, a_{i}\right)}(z)\right\}=\min \left\{1, \nu_{\left(f_{2}, a_{i}\right)}(z)\right\}$ for all $z \in \mathbb{C}^{m}$,

(ii) $\operatorname{dim}\left\{z:\left(f_{1}, a_{i}\right)(z)=\left(f_{1}, a_{i}\right)(z)=0\right\} \leq m-2$ for all $i \neq j$,

(iii) $f_{1}(z)=f_{2}(z)$ for all $z \in \bigcup_{i=1}^{q}\left(f_{1}, a_{i}\right)^{-1}(\{0\})$.

If $q>2 n^{2}+4 n$, then $f_{1} \equiv f_{2}$.

Here, we would like to note that in Corollaries 1.3 and 1.4 we do not need the condition on the nondegeneracy of the mappings. With the additional assumption that the mappings are linearly nondegenerate over $\mathcal{R}\left(\left\{a_{i}\right\}\right)$, Thai-Quang [10] proved the uniqueness theorem for the mappings sharing $q=2 n^{2}+4 n(n \geq 2)$. Without the assumption on the nondegeneracy, in 2007 Chen-Li-Yan 2 obtained the uniqueness theorem with $q=4 n^{2}+2 n(n \geq 2)$ and in 2013 Thoan-Duc-Quang 13 proved the uniqueness theorem for the case where $q>4 n^{2}+2$. Therefore, we see that Corollary 1.4 is an improvement of these results.

\section{BASIC NOTIONS AND AUXILIARY RESUlts FROM NEVANLINNA THEORY}

(a) Counting function of divisor.

For $z=\left(z_{1}, \ldots, z_{m}\right) \in \mathbb{C}^{m}$, we set $\|z\|=\left(\sum_{j=1}^{m}\left|z_{j}\right|^{2}\right)^{1 / 2}$ and define

$$
\begin{aligned}
B(r) & =\left\{z \in \mathbb{C}^{m} ;\|z\|<r\right\}, \quad S(r)=\left\{z \in \mathbb{C}^{m} ;\|z\|=r\right\}, \\
d^{c} & =\frac{\sqrt{-1}}{4 \pi}(\bar{\partial}-\partial), \quad \sigma=\left(d d^{c}\|z\|^{2}\right)^{m-1}, \\
\eta & =d^{c} \log \|z\|^{2} \wedge\left(d d^{c} \log \|z\|\right)^{m-1} .
\end{aligned}
$$

A divisor $E$ on $\mathbb{C}^{m}$ is given by a formal sum $E=\sum \mu_{\nu} X_{\nu}$, where $\left\{X_{\nu}\right\}$ is a locally family of distinct irreducible analytic hypersurfaces in $\mathbb{C}^{m}$ and $\mu_{\nu} \in \mathbb{Z}$. We define the support of the divisor $E$ by setting $\operatorname{Supp}(E)=\bigcup_{\nu \neq 0} X_{\nu}$. Sometimes we identify the divisor $E$ with a function $E(z)$ from $\mathbb{C}^{m}$ into $\mathbb{Z}$ defined by $E(z):=\sum_{X_{\nu} \ni z} \mu_{\nu}$.

Let $k$ be a positive integer or $+\infty$. We define the truncated divisor $E^{[k]}$ by

$$
E^{[k]}:=\sum_{\nu} \min \left\{\mu_{\nu}, k\right\} X_{\nu}
$$

and the truncated counting function to level $k$ of $E$ by

$$
N^{[k]}(r, E):=\int_{1}^{r} \frac{n^{[k]}(t, E)}{t^{2 m-1}} d t \quad(1<r<+\infty),
$$

where

$$
n^{[k]}(t, E):= \begin{cases}\int_{\operatorname{Supp}(E) \cap B(t)} E^{[k]} \sigma & \text { if } m \geq 2, \\ \sum_{|z| \leq t} E^{[k]}(z) & \text { if } m=1 .\end{cases}
$$

We omit the character ${ }^{[k]}$ if $k=+\infty$.

For an analytic hypersurface $E$ of $\mathbb{C}^{m}$, we may consider it as a reduced divisor and denote by $N(r, E)$ its counting function. 
Let $\varphi$ be a nonzero meromorphic function on $\mathbb{C}^{m}$. We denote by $\nu_{\varphi}^{0}$ (resp. $\nu_{\varphi}^{\infty}$ ) the divisor of zeros (resp. divisor of poles) of $\varphi$. The divisor of $\varphi$ is defined by

$$
\nu_{\varphi}=\nu_{\varphi}^{0}-\nu_{\varphi}^{\infty}
$$

We have the following Jensen's formula:

$$
N\left(r, \nu_{\varphi}^{0}\right)-N\left(r, \nu_{\varphi}^{\infty}\right)=\int_{S(r)} \log |\varphi| \eta-\int_{S(1)} \log |\varphi| \eta .
$$

For convenience, we will write $N_{\varphi}(r)$ and $N_{\varphi}^{[k]}(r)$ for $N\left(r, \nu_{\varphi}^{0}\right)$ and $N^{[k]}\left(r, \nu_{\varphi}^{0}\right)$, respectively.

(b) The first main theorem.

Let $f$ be a meromorphic mapping of $\mathbb{C}^{m}$ into $\mathbb{P}^{n}(\mathbb{C})$. For arbitrary fixed homogeneous coordinates $\left(w_{0}: \cdots: w_{n}\right)$ of $\mathbb{P}^{n}(\mathbb{C})$, we take a reduced representation $f=\left(f_{0}: \cdots: f_{n}\right)$, which means that each $f_{i}$ is a holomorphic function on $\mathbb{C}^{m}$ and $f(z)=\left(f_{0}(z): \cdots: f_{n}(z)\right)$ outside the analytic set $I(f):=\left\{z ; f_{0}(z)=\cdots=\right.$ $\left.f_{n}(z)=0\right\}$ of codimension at least 2 .

Denote by $\Omega$ the Fubini-Study form of $\mathbb{P}^{n}(\mathbb{C})$. The characteristic function of $f$ (with respect to $\Omega$ ) is defined by

$$
T_{f}(r):=\int_{1}^{r} \frac{d t}{t^{2 m-1}} \int_{B(t)} f^{*} \Omega \wedge \sigma, \quad 1<r<+\infty .
$$

By Jensen's formula we have

$$
T_{f}(r)=\int_{S(r)} \log \|f\| \eta+O(1)
$$

where $\|f\|=\max \left\{\left|f_{0}\right|, \ldots,\left|f_{n}\right|\right\}$.

Let $a$ be a meromorphic mapping of $\mathbb{C}^{m}$ into $\mathbb{P}^{n}(\mathbb{C})^{*}$ with a reduced representation $a=\left(a_{0}: \cdots: a_{n}\right)$. We define

$$
m_{f, a}(r)=\int_{S(r)} \log \frac{\|f\| \cdot\|a\|}{|(f, a)|} \eta-\int_{S(1)} \log \frac{\|f\| \cdot\|a\|}{|(f, a)|} \eta,
$$

where $\|a\|=\left(\left|a_{0}\right|^{2}+\cdots+\left|a_{n}\right|^{2}\right)^{1 / 2}$ and $(f, a)=\sum_{i=0}^{n} f_{i} \cdot a_{i}$.

Let $f$ and $a$ be as above. If $(f, a) \not \equiv 0$, then the first main theorem for moving hyperplanes in the value distribution theory states

$$
T_{f}(r)+T_{a}(r)=m_{f, a}(r)+N_{(f, a)}(r)+O(1)(r>1) .
$$

The mapping $a$ is usually called the moving hyperplanes. We will say that $a$ is slow with respect to $f$ if

$$
\| T_{a}(r)=o\left(T_{f}(r)\right) \text { as } a \longrightarrow+\infty .
$$

(c) Family of moving hyperplanes.

We assume that thoughout this paper, the homogeneous coordinates of $\mathbb{P}^{n}(\mathbb{C})$ are chosen so that for each given meromorphic mapping $a=\left(a_{0}: \cdots: a_{n}\right)$ of $\mathbb{C}^{m}$ into $\mathbb{P}^{n}(\mathbb{C})^{*}$, then $a_{0} \not \equiv 0$. We set

$$
\tilde{a}_{i}=\frac{a_{i}}{a_{0}} \text { and } \tilde{a}=\left(\tilde{a}_{0}: \tilde{a}_{1}: \cdots: \tilde{a}_{n}\right) \text {. }
$$

Let $f: \mathbb{C}^{m} \rightarrow \mathbb{P}^{n}(\mathbb{C})$ be a meromorphic mapping with the reduced representation $f=\left(f_{0}: \cdots: f_{n}\right)$. We put $(f, a):=\sum_{i=0}^{n} f_{i} a_{i}$ and $(f, \tilde{a}):=\sum_{i=0}^{n} f_{i} \tilde{a}_{i}$. 
Let $\left\{a_{i}\right\}_{i=1}^{q}$ be $q$ meromorphic mappings of $\mathbb{C}^{m}$ into $\mathbb{P}^{n}(\mathbb{C})^{*}$ with reduced representations $a_{i}=\left(a_{i 0}: \cdots: a_{i n}\right)(1 \leq i \leq q)$. We denote by $\mathcal{R}\left(\left\{a_{i}\right\}\right)$ (for brevity we will write $\mathcal{R}$ if there is no confusion) the smallest subfield of $\mathcal{M}$ which contains $\mathbb{C}$ and all $a_{i_{j}} / a_{i_{k}}$ with $a_{i_{k}} \neq \equiv 0$.

Definition 2.1. The family $\left\{a_{i}\right\}_{i=1}^{q}$ is said to be in general position if $\operatorname{dim}\left(\left\{a_{i_{0}}, \ldots, a_{i_{n}}\right\}\right)_{\mathcal{M}}=n+1$ for any $1 \leq i_{0} \leq \cdots \leq i_{n} \leq q$, where $\left(\left\{a_{i_{0}}, \ldots, a_{i_{n}}\right\}\right)_{\mathcal{M}}$ is the linear span of $\left\{a_{i_{0}}, \ldots, a_{i_{N}}\right\}$ over the field $\mathcal{M}$.

Definition 2.2. A subset $\mathcal{L}$ of $\mathcal{M}$ (or $\mathcal{M}^{n+1}$ ) is said to be minimal over the field $\mathcal{R}$ if it is linearly dependent over $\mathcal{R}$ and each proper subset of $\mathcal{L}$ is linearly independent over $\mathcal{R}$.

(d) Theorems for general position.

Theorem 2.3 (The First Main Theorem for general position [15, p. 326]). Let $f_{i}: \mathbb{C}^{m} \rightarrow \mathbb{P}^{n}(\mathbb{C}), 1 \leq i \leq k$, be meromorphic mappings located in general position. Assume that $1 \leq k \leq n$. Then

$$
N_{\mu_{f_{1} \wedge \cdots \wedge f_{\lambda}}}(r)+m\left(r, f_{1} \wedge \cdots \wedge f_{\lambda}\right) \leq \sum_{1 \leq i \leq \lambda} T_{f_{i}}(r)+O(1)
$$

Here, by $\mu_{f_{1} \wedge \cdots \wedge f_{\lambda}}$ we denote the divisor associated to $f_{1} \wedge \cdots \wedge f_{\lambda}$.

Let $V$ be a complex vector space of dimension $N \geq 1$. The vectors $\left\{v_{1}, \cdots, v_{k}\right\}$ are said to be in general position if for each selection of integers $1 \leq i_{1}<\cdots<$ $i_{p} \leq k$ with $p \leq N$, then $v_{i_{1}} \wedge \cdots \wedge v_{i_{p}} \neq 0$. The vectors $\left\{v_{1}, \cdots, v_{k}\right\}$ are said to be in special position if they are not in general position. Take $1 \leq p \leq k$. Then $\left\{v_{1}, \cdots, v_{k}\right\}$ are said to be in $p$-special position if for each selection of integers $1 \leq i_{1}<\cdots<i_{p} \leq k$, the vectors $v_{i_{1}}, \cdots, v_{i_{p}}$ are in special position.

Theorem 2.4 (The Second Main Theorem for general position [15, Theorem 2.1, p. 320]). Let $M$ be a connected complex manifold of dimension $m$. Let $A$ be a pure $(m-1)$-dimensional analytic subset of $M$. Let $V$ be a complex vector space of dimension $n+1>1$. Let $p$ and $k$ be integers with $1 \leq p \leq k \leq n+1$. Let $f_{i}: M \rightarrow P(V), 1 \leq i \leq k$, be meromorphic mappings. Assume that $f_{1}, \ldots, f_{k}$ are in general position. Also assume that $f_{1}, \ldots, f_{k}$ are in $p$-special position on $A$. Then we have

$$
\mu_{f_{1} \wedge \cdots \wedge f_{k}} \geq(k-p+1) \nu_{A}
$$

\section{Proof of Theorem 1.1}

In order to prove Theorem 1.1 we need the following.

Lemma 3.1 (see [5, Lemma 3.1]). Let $f: \mathbb{C}^{m} \rightarrow \mathbb{P}^{n}(\mathbb{C})$ be a meromorphic mapping. Let $\left\{a_{i}\right\}_{i=1}^{q}(q \geq n+1)$ be $q$ meromorphic mappings of $\mathbb{C}^{m}$ into $\mathbb{P}^{n}(\mathbb{C})^{*}$ in general position. Assume that there exists a partition $\{1, \ldots, q\}=I_{1} \cup I_{2} \cup \cdots \cup I_{l}$ satisfying:

(i) $\left\{\left(f, \tilde{a}_{i}\right)\right\}_{i \in I_{1}}$ is minimal over $\mathcal{R}$, and $\left\{\left(f, \tilde{a}_{i}\right)\right\}_{i \in I_{t}}$ is linearly independent over $\mathcal{R}(2 \leq t \leq l)$.

(ii) For any $2 \leq t \leq l, i \in I_{t}$, there exist meromorphic functions $c_{i} \in \mathcal{R} \backslash\{0\}$ such that

$$
\sum_{i \in I_{t}} c_{i}\left(f, \tilde{a}_{i}\right) \in\left(\bigcup_{j=1}^{t-1} \bigcup_{i \in I_{j}}\left(f, \tilde{a}_{i}\right)\right)_{\mathcal{R}} .
$$


Then we have

$$
T_{f}(r) \leq \sum_{i=1}^{q} N_{\left(f, a_{i}\right)}^{[k]}+o\left(T_{f}(r)\right)+O\left(\max _{1 \leq i \leq q} T_{a_{i}}(r)\right),
$$

where $k+1=\operatorname{rank}_{\mathcal{R}}(f)$.

Proof of Theorem 1.1 .

(a) We denote by $\mathcal{I}$ the set of all permutations of $q$-tuple $(1, \ldots, q)$. For each element $I=\left(i_{1}, \ldots, i_{q}\right) \in \mathcal{I}$, we set

$$
N_{I}=\left\{r \in \mathbf{R}^{+} ; N_{\left(f, a_{i_{1}}\right)}^{[k]}(r) \leq \cdots \leq N_{\left(f, a_{i_{q}}\right)}^{[k]}(r)\right\} .
$$

We now consider an element $I=\left(i_{1}, \ldots, i_{q}\right)$ of $\mathcal{I}$. We will construct subsets $I_{t}$ of the set $A_{1}=\{1, \ldots, 2 n-k+2\}$ as follows.

We choose a subset $I_{1}$ of $A$ which is the minimal subset of $A$ satisfying that $\left\{\left(f, \tilde{a}_{i_{j}}\right)\right\}_{j \in I_{1}}$ is minimal over $\mathcal{R}$. If $\sharp I_{1} \geq n+1$, then we stop the process.

Otherwise, set $A_{2}=A_{1} \backslash I_{1}$. We consider the following two cases:

- Case 1. Suppose that $\sharp A_{2} \geq n+1$. Since $\left\{\tilde{a}_{i_{j}}\right\}_{j \in A_{2}}$ is in general position, we have

$$
\left(\left(f, \tilde{a}_{i_{j}}\right) ; j \in A_{2}\right)_{\mathcal{R}}=\left(f_{0}, \ldots, f_{n}\right)_{\mathcal{R}} \supset\left(\left(f, \tilde{a}_{i_{j}}\right) ; j \in I_{1}\right)_{\mathcal{R}} \not \equiv 0 .
$$

- Case 2. Suppose that $\sharp A_{2}<n+1$. Then we have the following:

$$
\begin{aligned}
& \operatorname{dim}_{\mathcal{R}}\left(\left(f, \tilde{a}_{i_{j}}\right) ; j \in I_{1}\right)_{\mathcal{R}} \geq k+1-\left(n+1-\sharp I_{1}\right)=k-n+\sharp I_{1}, \\
& \operatorname{dim}_{\mathcal{R}}\left(\left(f, \tilde{a}_{i_{j}}\right) ; j \in A_{2}\right)_{\mathcal{R}} \geq k+1-\left(n+1-\sharp A_{2}\right)=k-n+\sharp A_{2} .
\end{aligned}
$$

We note that $\sharp I_{1}+\sharp A_{2}=2 n-k+2$. Hence the above inequalities imply that

$$
\begin{aligned}
\operatorname{dim}_{\mathcal{R}} & \left(\left(\left(f, \tilde{a}_{i_{j}}\right) ; j \in I_{1}\right)_{\mathcal{R}} \cap\left(\left(f, \tilde{a}_{i_{j}}\right) ; j \in A_{2}\right)_{\mathcal{R}}\right) \\
& \geq \operatorname{dim}_{\mathcal{R}}\left(\left(f, \tilde{a}_{i_{j}}\right) ; j \in I_{1}\right)_{\mathcal{R}}+\operatorname{dim}_{\mathcal{R}}\left(\left(f, \tilde{a}_{i_{j}}\right) ; j \in A_{2}\right)_{\mathcal{R}}-(k+1) \\
& =k-n+\sharp I_{1}+k-n+\sharp A_{2}-(k+1)=1 .
\end{aligned}
$$

Therefore, from the above two cases, we see that

$$
\left(\left(f, \tilde{a}_{i_{j}}\right) ; j \in I_{1}\right)_{\mathcal{R}} \cap\left(\left(f, \tilde{a}_{i_{j}}\right) ; j \in A_{2}\right)_{\mathcal{R}} \neq\{0\} .
$$

Therefore, we may choose a subset $I_{2} \subset A_{2}$ which is the minimal subset of $A_{2}$ satisfying that there exist nonzero meromorphic functions $c_{i} \in \mathcal{R}\left(i \in I_{2}\right)$ so that

$$
\sum_{i \in I_{2}} c_{i}\left(f, \tilde{a}_{i}\right) \in\left(\bigcup_{i \in I_{1}}\left(f, \tilde{a}_{i}\right)\right)_{\mathcal{R}} .
$$

By the minimality of the set $I_{2}$, the family $\left\{\left(f, \tilde{a}_{i_{j}}\right)\right\}_{j \in I_{2}}$ is linearly independent over $\mathcal{R}$, and hence $\sharp I_{2} \leq k+1$ and

$$
\sharp\left(I_{2} \cup I_{2}\right) \leq 2 n-k+2 .
$$

If $\sharp\left(I_{2} \cup I_{2}\right) \geq n+1$, then we stop the process. 
Otherwise, by repeating the above argument, we have a subset $I_{3}$ of $A_{3}=A_{1} \backslash$ $\left(I_{1} \cup I_{2}\right)$, which satisfies the following:

- there exist nonzero meromorphic functions $c_{i} \in \mathcal{R}\left(i \in I_{3}\right)$ so that

$$
\sum_{i \in I_{3}} c_{i}\left(f, \tilde{a}_{i}\right) \in\left(\bigcup_{i \in I_{1} \cup I_{2}}\left(f, \tilde{a}_{i}\right)\right)_{\mathcal{R}},
$$

- $\left\{\left(f, \tilde{a}_{i_{j}}\right)\right\}_{j \in I_{3}}$ is linearly independent over $\mathcal{R}$.

Continuing this process, we get the subsets $I_{1}, \ldots, I_{l}$, which satisfy:

- $\left\{\left(f, \tilde{a}_{i_{j}}\right)\right\}_{j \in I_{1}}$ is minimal over $\mathcal{R}$ and $\left\{\left(f, \tilde{a}_{i_{j}}\right)\right\}_{j \in I_{t}}$ is linearly independent over $\mathcal{R}(2 \leq t \leq l)$,

- for any $2 \leq t \leq l, j \in I_{t}$, there exist meromorphic functions $c_{j} \in \mathcal{R} \backslash\{0\}$ such that

$$
\sum_{j \in I_{t}} c_{j}\left(f, \tilde{a}_{i_{j}}\right) \in\left(\bigcup_{s=1}^{t-1} \bigcup_{j \in I_{s}}\left(f, \tilde{a}_{i_{j}}\right)\right)_{\mathcal{R}} .
$$

Then the family of subsets $I_{1}, \ldots, I_{t}$ satisfies the assumptions of Lemma 3.1 . Therefore, we have

$$
\begin{aligned}
\| T_{f}(r) & \leq \sum_{j \in I_{1} \cup \cdots \cup I_{l}} N_{\left(f, a_{i_{j}}\right)}^{[k]}+o\left(T_{f}(r)\right)+O\left(\max _{1 \leq i \leq q} T_{a_{i}}(r)\right) \\
& \leq \sum_{j=1}^{2 n-k+2} N_{\left(f, a_{i_{j}}\right)}^{[k]}+o\left(T_{f}(r)\right)+O\left(\max _{1 \leq i \leq q} T_{a_{i}}(r)\right) .
\end{aligned}
$$

Then for all $r \in N_{I}$ (maybe outside a finite Borel measure subset of $\mathbf{R}^{+}$) we have

$$
\begin{aligned}
\|\left(\sum_{i=1}^{q} \lambda_{i}\right) T_{f}(r) \leq & \left(\sum_{i=1}^{q} \lambda_{i}\right)\left(\sum_{j=1}^{2 n-k+2} N_{\left(f, a_{i_{j}}\right)}^{[k]}(r)\right)+o\left(T_{f}(r)\right)+O\left(\max _{1 \leq i \leq q} T_{a_{i}}(r)\right) \\
\leq & (2 n-k+2)\left(\sum_{j=1}^{2 n-k+2} \frac{\sum_{i=1}^{q} \lambda_{i}}{2 n-k+2} N_{\left(f, a_{i_{j}}\right)}^{[k]}(r)\right) \\
+ & o\left(T_{f}(r)\right)+O\left(\max _{1 \leq i \leq q} T_{a_{i}}(r)\right) \\
= & (2 n-k+2) \sum_{j=1}^{2 n-k+2}\left(\lambda_{i_{j}} N_{\left(f, a_{i_{j}}\right)}^{[k]}(r)\right. \\
& \left.+\sum_{j=1}^{2 n-k+2}\left(\frac{\sum_{i=1}^{q} \lambda_{i}}{2 n-k+2} \lambda_{i_{j}}\right) N_{\left(f, a_{i_{j}}\right)}^{[k]}(r)\right)+o\left(T_{f}(r)\right)+O\left(\max _{1 \leq i \leq q} T_{a_{i}}(r)\right) \\
\leq & (2 n-k+2) \sum_{j=1}^{2 n-k+2} \lambda_{i_{j}} N_{\left(f, a_{i_{j}}\right)}^{[k]}(r) \\
& \sum_{j=}^{q} \lambda_{i_{j}} N_{\left(f, a_{i_{2 n-k+2}}^{[k]}\right.}^{[k+k+3}(r)+o\left(T_{f}(r)\right)+O\left(\max _{1 \leq i \leq q} T_{a_{i}}(r)\right) \\
\leq & (2 n-k+2) \sum_{j=1}^{q} \lambda_{i_{j}} N_{\left(f, a_{i_{j}}\right)}^{[k]}(r)+o\left(T_{f}(r)\right)+O\left(\max _{1 \leq i \leq q} T_{a_{i}}(r)\right) .
\end{aligned}
$$


Then we have

$$
\| \frac{\sum_{i=1}^{q} \lambda_{i}}{2 n-k+2} T_{f}(r) \leq \sum_{i=1}^{q} \lambda_{i} N_{\left(f, a_{i}\right)}^{[k]}(r)+o\left(T_{f}(r)\right)+O\left(\max _{1 \leq i \leq q} T_{a_{i}}(r)\right), \quad r \in N_{I} .
$$

We see that $\bigcup_{I \in \mathcal{I}} N_{I}=\mathbf{R}^{+}$and the inequality (3.2) holds for every $r \in N_{I}, I \in \mathcal{I}$. This yields that

$$
\| \frac{\sum_{i=1}^{q} \lambda_{i}}{2 n-k+2} T_{f}(r) \leq \sum_{i=1}^{q} \lambda_{i} N_{\left(f, a_{i}\right)}^{[k]}(r)+o\left(T_{f}(r)\right)+O\left(\max _{1 \leq i \leq q} T_{a_{i}}(r)\right) .
$$

The theorem is proved.

\section{Proof of Theorem 1.2}

In order to prove Theorem 1.2, we need the following lemma.

Lemma 4.1. With the assumptions of Theorem 1.1, we have

$$
\| \frac{\sum_{i=1}^{q} \lambda_{i}}{n^{2}+2 n} T_{f}(r) \leq \sum_{i=1}^{q} \lambda_{i} N_{\left(f, a_{i}\right)}^{[1]}(r)+o\left(T_{f}(r)\right)+O\left(\max _{1 \leq i \leq q} T_{a_{i}}(r)\right) .
$$

Proof. From Theorem 1.1 we have

$$
\begin{aligned}
\| \sum_{i=1}^{q} \lambda_{i} N_{\left(f, a_{i}\right)}^{[1]}(r) & \geq \frac{1}{k} \sum_{i=1}^{q} \lambda_{i} N_{\left(f, a_{i}\right)}^{[k]}(r) \\
& \geq \frac{\sum_{i=1}^{q} \lambda_{i}}{(2 n-k+2) k} T_{f}(r)+o\left(T_{f}(r)\right)+O\left(\max _{1 \leq i \leq q} T_{a_{i}}(r)\right) \\
& \geq \frac{\sum_{i=1}^{q} \lambda_{i}}{n^{2}+2 n} T_{f}(r)+o\left(T_{f}(r)\right)+O\left(\max _{1 \leq i \leq q} T_{a_{i}}(r)\right) .
\end{aligned}
$$

Here we note that $(2 n-k+2) k=(n+1)^{2}-(n+1-k)^{2} \leq(n+1)^{2}-1=n^{2}+2 n$, since $k \leq n$. Then the lemma is proved.

It suffices to prove Theorem 1.2 in the case of $\lambda \leq N+1$.

Assume that $f_{1} \wedge \cdots \wedge f_{\lambda} \not \equiv 0$. We denote by $\mu_{f_{1} \wedge \cdots \wedge f_{\lambda}}$ the divisor associated with $f_{1} \wedge \cdots \wedge f_{\lambda}$. Denote by $N_{f_{1} \wedge \cdots \wedge f_{\lambda}}(r)$ the counting function associated with the divisor $\mu_{f_{1} \wedge \cdots \wedge f_{\lambda}}$. We now prove the following.

Claim 4.2. For every $1 \leq t \leq \lambda$, we have

$\sum_{j=1}^{q}\left(\lambda-l_{j}+1\right) \min \left\{1, \nu_{\left(f_{t}, g_{j}\right)}(z)\right\} \leq d \mu_{f_{1} \wedge \cdots \wedge f_{\lambda}}(z)+q(\lambda-1) \sum_{\beta} \mu_{g_{\beta(1)} \wedge \cdots \wedge g_{\beta(n+1)}}(z)$

for each $z \notin A \cup \bigcup_{i=1}^{\lambda} I\left(f_{i}\right)$, where the sum is taken over all injective maps $\beta$ : $\{1,2, \cdots, n+1\} \rightarrow\{1,2, \cdots, q\}$

We now prove Claim 4.2. Set $\mathcal{A}=\bigcup_{j=1}^{q}\left(f_{t}, g_{j}\right)^{-1}\{0\}$. For each regular point $z_{0} \in \mathcal{A} \backslash\left(A \cup \bigcup_{i=1}^{\lambda} I\left(f_{i}\right) \cup \bigcup_{\beta \in T[n+1, q]}\left\{z \mid g_{\beta(1)}(z) \wedge \cdots \wedge g_{\beta(N+1)}(z)=0\right\}\right)$ and for each increasing sequence $1 \leq j_{1}<\cdot<j_{l} \leq \lambda$, we have

$$
f_{j_{1}}\left(z_{0}\right) \wedge \cdots \wedge f_{j_{l}}\left(z_{0}\right)=0
$$

where $l=\max _{z_{0} \in A_{j}} l_{j}$. 
By the Second Main Theorem for general position [15, Theorem 2.1, p. 320], we have

$$
\mu_{f_{1} \wedge \cdots \wedge f_{\lambda}}\left(z_{0}\right) \geq \lambda-(l-1)
$$

Hence

$$
\sum_{j=1}^{q} \min \left\{1, \nu_{\left(f_{t}, g_{j}\right)}\left(z_{0}\right)\right\} \leq d \leq \frac{d}{\lambda-l+1} \mu_{f_{1} \wedge \cdots \wedge f_{\lambda}}\left(z_{0}\right) .
$$

This implies that

$$
\sum_{j=1}^{q}\left(\lambda-l_{j}+1\right) \min \left\{1, \nu_{\left(f_{t}, g_{j}\right)}\left(z_{0}\right)\right\} \leq d \mu_{f_{1} \wedge \cdots \wedge f_{\lambda}}\left(z_{0}\right) .
$$

If $z_{0} \in \bigcup_{\beta \in T[n+1, q]}\left\{z \mid g_{\beta(1)}(z) \wedge \cdots \wedge g_{\beta(n+1)}(z)=0\right\}$, then we have

$$
\left(\lambda-l_{j}+1\right) \sum_{j=1}^{q} \min \left\{1, \nu_{\left(f_{t}, g_{j}\right)}\left(z_{0}\right)\right\} \leq(\lambda-1) q \sum_{\beta \in T[n+1, q]} \mu_{g_{\beta(1)} \wedge \cdots \wedge g_{\beta(n+1)}}\left(z_{0}\right) .
$$

Thus, for each $z \notin A \cup \bigcup_{j=1}^{\lambda} I\left(f_{j}\right)$, we have

$$
\begin{aligned}
\sum_{j=1}^{q}\left(\lambda-l_{j}+1\right) \min \left\{1, \nu_{\left(f_{t}, g_{j}\right)}(z)\right\} \leq & d \mu_{f_{1} \wedge \cdots \wedge f_{\lambda}}(z) \\
& +(\lambda-1) q \sum_{\beta \in T[n+1, q]} \mu_{g_{\beta(1)} \wedge \cdots \wedge g_{\beta(n+1)}}(z) .
\end{aligned}
$$

Then Claim 4.2 is proved.

The above claim yields that

$$
\begin{aligned}
\sum_{j=1}^{q}\left(\lambda-l_{j}+1\right) N_{\left(f_{t}, g_{j}\right)}^{[1]}(r) & \leq d N_{\mu_{f_{1} \wedge \cdots \wedge f_{\lambda}}}(r)+(\lambda-1) q N \sum_{\beta \in T[n+1, q]} N_{\mu_{g_{\beta(1)} \wedge \cdots \wedge g_{\beta(n+1)}}}(r) \\
& \leq d \sum_{i=1}^{\lambda} T_{f_{i}}(r)+q N \sum_{\beta \in T[n+1, q]} \sum_{i=1}^{n+1} T_{a_{\beta(i)}}(r) \\
& =d \sum_{i=1}^{\lambda} T_{f_{i}}(r)+o\left(\max _{1 \leq i \leq \lambda} T_{f_{i}}(r)\right) .
\end{aligned}
$$

Thus, by summing up both sides of these inequalities, we have

$$
\sum_{t=1}^{\lambda} \sum_{j=1}^{q}\left(\lambda-l_{j}+1\right) N_{\left(f_{t}, g_{j}\right)}^{[1]}(r) \leq d \lambda \sum_{i=1}^{\lambda} T_{f_{i}}(r)+o\left(\max _{1 \leq i \leq \lambda} T_{f_{i}}(r) .\right.
$$

We easily see that

$$
\left(2 n-\operatorname{rank}_{\mathcal{R}\left\{a_{i}\right\}}\left(f_{t}\right)+3\right) \max _{1 \leq j \leq q}\left(\lambda-l_{j}+1\right) \leq(2 n+1)(\lambda-1) \leq q \leq \sum_{j=1}^{q}\left(\lambda-l_{j}+1\right) .
$$

Then by using Lemma 4.1, the inequality (4.3) implies that

$$
\| \sum_{i=1}^{\lambda} \frac{q(\lambda+1)-\sum_{j=1}^{q} l_{j}}{n^{2}+2 n} T_{f_{i}}(r) \leq d \lambda \sum_{i=1}^{\lambda} T_{f_{i}}(r)+o\left(\max _{1 \leq i \leq \lambda} T_{f_{i}}(r)\right) .
$$


Letting $r \rightarrow+\infty$, we get $q \leq \frac{d \lambda\left(n^{2}+2 n\right)+\sum_{j=1}^{q} l_{j}}{\lambda+1}$. This is a contradiction.

Thus, the family $\left\{f_{1}, \cdots, f_{\lambda}\right\}$ is algebraically dependent over $\mathbb{C}$, i.e., $f_{1} \wedge \cdots \wedge f_{\lambda}=$ 0 . Theorem 1.2 is proved.

\section{ACKNOWLEDGEMENTS}

The research of the author was supported by a NAFOSTED grant of Vietnam (Grant No. 101.04-2015.03).

\section{REFERENCES}

[1] H. Cartan, Sur les zeros des combinasons lineaires de p fonctions holomorphes donnees, Mathematica 7 (1933), 5-31.

[2] Zhihua Chen, Yanhua Li, and Qiming Yan, Uniqueness problem with truncated multiplicities of meromorphic mappings for moving targets, Acta Math. Sci. Ser. B Engl. Ed. 27 (2007), no. 3, 625-634, DOI 10.1016/S0252-9602(07)60062-9. MR2339402 (2008k:32047)

[3] I. E. Nochka, On the theory of meromorphic functions, Sov. Math. Dokl. 27 (1983), 377-381.

[4] Junjiro Noguchi and Takushiro Ochiai, Geometric function theory in several complex variables, Translations of Mathematical Monographs, vol. 80, American Mathematical Society, Providence, RI, 1990. Translated from the Japanese by Noguchi. MR,1084378 (92e:32001)

[5] S. D. Quang, Second main theorems for meromorphic mappings intersecting moving hyperplanes with truncated counting functions and unicity problem, Abh. Math. Semin. Univ. Hambg. (2015) pp 1-18, DOI: 10.1007/s12188-015-0114-1.

[6] Si Duc Quang, Algebraic dependences of meromorphic mappings sharing few moving hyperplanes, Ann. Polon. Math. 108 (2013), no. 1, 61-73, DOI 10.4064/ap108-1-5. MR3021268

[7] Si Duc Quang and Do Phuong An, Unicity of meromorphic mappings sharing few moving hyperplanes, Vietnam J. Math. 41 (2013), no. 4, 383-398, DOI 10.1007/s10013-013-0035-1. MR3142403

[8] Min Ru, A uniqueness theorem with moving targets without counting multiplicity, Proc. Amer. Math. Soc. 129 (2001), no. 9, 2701-2707, DOI 10.1090/S0002-9939-01-06040-3. MR.1838794 (2002e:32024)

[9] Min Ru and Julie Tzu-Yueh Wang, Truncated second main theorem with moving targets, Trans. Amer. Math. Soc. 356 (2004), no. 2, 557-571 (electronic), DOI 10.1090/S0002-994703-03453-6. MR2022710 (2004j:32018)

[10] Do Duc Thai and Si Duc Quang, Uniqueness problem with truncated multiplicities of meromorphic mappings in several complex variables for moving targets, Internat. J. Math. 16 (2005), no. 8, 903-939, DOI 10.1142/S0129167X05003132. MR2168074 (2006h:32014)

[11] Duc Thai Do and Duc Quang Si, Second main theorem with truncated counting function in several complex variables for moving targets, Forum Math. 20 (2008), no. 1, 163-179, DOI 10.1515/FORUM.2008.007. MR2386785 (2009a:32021)

[12] Duc Thoan Pham and Viet Duc Pham, Algebraic dependences of meromorphic mappings in several complex variables, Ukrainian Math. J. 62 (2010), no. 7, 1073-1089, DOI 10.1007/s11253-010-0414-7. MR2888660

[13] Pham Duc Thoan, Pham Viet Duc, and Si Duc Quang, Algebraic dependence and unicity theorem with a truncation level to 1 of meromorphic mappings sharing moving targets, Bull. Math. Soc. Sci. Math. Roumanie (N.S.) 56(104) (2013), no. 4, 513-526. MR3157097

[14] Bernard Shiffman, Introduction to the Carlson-Griffiths equidistribution theory, Value distribution theory (Joensuu, 1981), Lecture Notes in Math., vol. 981, Springer, Berlin-New York, 1983, pp. 44-89. MR699133 (84j:32033)

[15] Wilhelm Stoll, On the propagation of dependences, Pacific J. Math. 139 (1989), no. 2, 311337. MR1011216(91a:32037) 
[16] Katsutoshi Yamanoi, The second main theorem for small functions and related problems, Acta Math. 192 (2004), no. 2, 225-294, DOI 10.1007/BF02392741. MR2096455 (2006g:30050)

Department of Mathematics, Hanoi National University of Education, 136-Xuan Thuy, Cau Giay, Hanoi, Vietnam

E-mail address: quangsd@hnue.edu.vn 